\title{
A SINGULAR SPACE RELATED TO THE POINT-OPEN GAME
}

\author{
H. J. K. JUNNILA, T. NOGURA AND R. TELGÁRSKY
}

\begin{abstract}
The authors construct a completely regular space $X_{0}$ such that Player I has a winning strategy in the point-open game $G\left(X_{0}\right)$, but $X_{0}$ has no $\sigma$-closure-preserving cover by $\mathrm{C}$-scattered closed sets.
\end{abstract}

The purpose of this paper is to present the following.

EXAMPLE. A completely regular space $X_{0}$ such that Player I has a winning strategy in the point-open game $G\left(X_{0}\right)$, but $X_{0}$ has no $\sigma$-closure-preserving cover by $\mathbf{C}$-scattered closed sets.

Given a space $X$, the point-open game $G(X)$ is defined as follows: at move $n$, Player I chooses a point $x_{n}$ in $X$, and then Player II chooses an open neighborhood $U_{n}$ of $x_{n}$. Player I wins the play $\left(x_{0}, U_{0}, x_{1}, U_{1}, \ldots\right)$ of $G(X)$ if $\bigcup\left\{U_{n}: n<\omega\right\}=X$ (see $\left[\mathbf{G}, \mathbf{T}_{2}\right]$ ). If instead of points $x_{n}$ Player I chooses compact sets $C_{n}$, and if Player I has a winning strategy in the resulting game, then $X$ is called compactlike (see $\left[\mathbf{T}_{2}, \mathbf{T}_{3}\right]$ ). If a point $x$ in a space $X$ has an open neighborhood $U$ such that $\bar{U}$ is compact, then $x$ is called a point of local compactness of $X$. A space $X$ such that each nonempty closed subspace has a point of local compactness is called $\mathbf{C}$-scattered $\left[\mathbf{T}_{1}\right]$. A family $\mathcal{F}$ of subsets of $X$ with the property that for any subfamily of $\mathcal{F}$, the closure and the sum commute, is called closure-preserving $[\mathbf{M}]$. A countable union of closure-preserving families is called $\sigma$-closure-preserving.

The space $X_{0}$ in the above example is a modification of the space constructed by Nogura $[\mathbf{N}]$; however, it improves his result in several aspects. Nogura showed, under the continuum hypotheses, that a compact-like space need not have a countable cover by $\mathbf{C}$-scattered closed subsets, answering a question of Telgársky $\left[\mathbf{T}_{2}, \mathbf{T}_{3}\right]$. Here, (1) the continuum hypothesis is eliminated, (2) the game condition involving Player I is substantially strengthened, and (3) the countable union is generalized to a $\sigma$-closure-preserving union.

Since each compact space is $\mathbf{C}$-scattered, it follows that $X_{0}$ has no $\sigma$-closurepreserving cover by compact sets. In contrast, a hereditarily metacompact compactlike space does have a closure-preserving cover by compact sets (see [JST]). Moreover, each compact-like space is the union of countably many C-scattered subsets (see $\left[\mathbf{T}_{3}\right]$ ), where the $\mathbf{C}$-scattered subsets cannot be closed in general (it follows from $[\mathbf{N}]$ and also from the above example).

Received by the editors December 18, 1985 and, in revised form, January 30, 1986 . The contents of this paper have been presented to the American Mathematical Society at its 92nd Annual Meeting in New Orleans, Louisiana, January 7-11, 1986 (Session on General Topology, \#569) by $\mathrm{R}$. Telgársky.

1980 Mathematics Subject Classification (1985 Revision). Primary 54D20, 90D42, 54D30, 54G20.

Key words and phrases. Point-open game, compact-like space, closure-preserving, $\sigma$-closurepreserving, C-scattered, metacompact. 
Construction. Let $T=\left\{t_{\alpha}: \alpha<\omega_{1}\right\}$ be a subset of the closed unit interval $[0,1]$, where $t_{\alpha} \neq t_{\beta}$ for $\alpha \neq \beta$. Let $T_{0}=T \times\left\{\omega_{1}\right\}$ and

$$
X=\left\{\left(t_{\alpha}, \beta\right): \alpha<\beta<\omega_{1}\right\} \cup T_{0} .
$$

The space $X$ is a subspace of $[0,1] \times\left[0, \omega_{1}\right]$, where $\left[0, \omega_{1}\right]$ has the standard interval topology (that is, the topology generated by left-open right-closed intervals). The space $X_{0}$ is obtained from $X$ by contracting the set $T_{0}$ into the singleton $\left\{x_{0}\right\}$.

Claim 1. Player I has a winning strategy in $G\left(X_{0}\right)$.

PROOF. Observe that the complement of any neighborhood of $x_{0}$ in $X_{0}$ is countable. Therefore, if the first move of Player $I$ is $x_{0}$, he can easily take care of the remaining countable set.

For a subset $H$ of $X_{0}$ and a $t \in T$ define

$$
H_{t}=\left\{\alpha<\omega_{1}:(t, \alpha) \in H\right\} .
$$

Claim 2. If $H$ is closed in $X_{0}$, then $H_{t}$ is closed in $\left[0, \omega_{1}\right)$.

This is immediate, since the map which takes $(t, \alpha)$ to $\alpha$ is a homeomorphism between a subspace of $X_{0}$ and a closed subspace of $\left[0, \omega_{1}\right)$.

For a subset $H$ of $X_{0}$ define

$$
T_{H}=\left\{t \in T: H_{t} \text { is uncountable }\right\} .
$$

Claim 3. If $H$ is closed in $X_{0}$, then $T_{H}$ is closed in $T$.

PROOF. Let $t=\lim t(n)$, where $t \in T$ and $t(n) \in T_{H}$ for each $n<\omega$. Then each $H_{t(n)}$ is uncountable and closed in $\left[0, \omega_{1}\right)$, and thus $E=\bigcap\left\{H_{t(n)}: n<\omega\right\}$ is also uncountable and closed in $\left[0, \omega_{1}\right)$. Take an $\alpha<\omega_{1}$ such that $t_{\alpha}=t$. Then $E \cap\left(\alpha, \omega_{1}\right) \subset H_{t}$, and hence $t \in T_{H}$.

ClaIM 4. If $\nLeftarrow$ is a $\sigma$-closure-preserving family of closed subsets of $X_{0}$, then $\bigcup\left\{T_{H}: H \in \mathcal{H}\right\}=T_{\cup \mathcal{H}}$.

ProOF. Let $t \in T_{\cup \mathcal{H}}$. Then $(\bigcup \mathcal{H})_{t}$ is uncountable and is covered by the $\sigma$ closure-preserving closed family $\left\{H_{t}: H \in \mathcal{H}\right\}$. By Corollary 1 of Potoczny and Junnila [PJ], if each $H_{t}$ were compact, then $(\bigcup \mathcal{K})_{t}$ would be the countable union of metacompact closed subsets of $\left[0, \omega_{1}\right)$. Since each metacompact closed subset of $\left[0, \omega_{1}\right)$ is countable, there is an $H \in \mathscr{H}$ such that $H_{t}$ is not compact. Hence $t \in T_{H}$.

From Claims 3 and 4 we get

Claim 5. If $\not$ is a $\sigma$-closure-preserving closed cover of $X_{0}$, then $\left\{T_{H}: H \in \not{H}\right\}$ is a $\sigma$-closure-preserving closed cover of $T$.

Let $\not$ be a $\sigma$-closure-preserving closed cover of $X_{0}$. We shall show in Claim 7 that some element of $\forall$ is not $\mathbf{C}$-scattered.

Claim 6. $T_{H}$ is uncountable for some $H \in \mathcal{H}$.

ProOF. Since $T$ is hereditarily separable, the $\sigma$-closure-preserving cover $\left\{T_{H}\right.$ : $H \in \mathscr{H}\}$ of $T$ has a countable subcover. Hence the claim follows.

Let $H$ be an element of $\nLeftarrow$ such that $T_{H}$ is uncountable.

Claim 7. $H$ is not C-scattered.

PROOF. Let $\{t(n): n<\omega\}$ be a countable self-dense subset of $T_{H}$. Each $H_{t(n)}$ is a closed unbounded set in $\left[0, \omega_{1}\right)$, hence there exists an $\alpha$ in $\bigcap\left\{H_{t(n)}: n<\omega\right\}$. Let $K$ be the closure of $\{(t(n), \alpha): n<\omega\}$ in $X_{0}$. Then $K$ is a countable self-dense closed subset of $H$. Hence $H$ is not $\mathbf{C}$-scattered. 


\section{REFERENCES}

[G] F. Galvin, Indeterminacy of point-open games, Bull. Acad. Polon. Sci. 26 (1978), 445-449.

[JST] H. J. K. Junnila, J. C. Smith and R. Telgársky, Closure-preserving covers by small sets, Topology Appl. 23 (1986), 237-262.

[M] E. Michael, Another note on paracompact spaces, Proc. Amer. Math. Soc. 8 (1957), 822-828.

[N] T. Nogura, A compact-like space which does not have a countable cover by $C$-scattered closed subsets, Proc. Japan Acad. 59 (1983), 83-84.

[PJ] H. Potoczny and H. Junnila, Closure-preserving families and metacompactness, Proc. Amer. Math. Soc. 53 (1975), 523-529.

$\left[\mathbf{T}_{1}\right]$ R. Telgársky, $C$-scattered and paracompact spaces, Fund. Math. 73 (1971), 59-74.

$\left[\mathbf{T}_{2}\right]$ _, Spaces defined by topological games, Fund. Math. 88 (1975), 193-223.

$\left[\mathbf{T}_{3}\right] \ldots$, Spaces defined by topological games, II, Fund. Math. 116 (1983), 189-207.

Department of Mathematics, University of Helsinki, 00100 Helsinki 10, FinLAND

Department of Mathematics, Ehime University, Matsuyama 790, JAPAN

Department of Mathematics, OHio University, ATHENS, OHIO 45701 